\title{
Partial transport of a sand/gravel sediment
}

\author{
Peter R. Wilcock and Brian W. McArdell \\ Department of Geography and Environmental Engineering, Johns Hopkins University, Baltimore, Maryland
}

\begin{abstract}
Grains of a single size within a mixed-size bed are entrained over a range of flows. Within this range some grains exposed on the bed surface are active (entrained at least once over the duration of a transport event), while the remaining surface grains are immobile, a condition we define as partial transport. We demonstrate the existence and domain of partial transport using observations of grain entrainment on time series of bed photographs of flume experiments with a widely sorted sand/gravel mixture. The active proportion of the bed surface increases with bed shear stress $\tau_{0}$. At a given $\tau_{0}, 90 \%$ of the active grains are entrained when the cumulative mass transported exceeds approximately 4 times the active mass on the bed. Mobilization of grains in a size fraction increases from $10 \%$ to $90 \%$ over a range of $\tau_{0}$ of a factor of 2 . The bounds of this range increase with grain size $D_{i}$ so that at a given $\tau_{0}$, sizes over a range of a factor of 4 are in a state of partial transport. Fractional transport rates are independent of $D_{i}$ for fully mobilized fractions and decrease rapidly with $D_{i}$ for partially mobile fractions. Partial transport is associated with substantial transport rates of finer, fully mobile sizes, limits both the rate and size distribution of grain exchange with the bed subsurface, and may be the dominant transport condition in many gravel-bed rivers.
\end{abstract}

\section{Introduction}

The size of sediment grains transported by gravel-bed rivers typically increases with flow strength. Over a range of flows with measurable transport, the transport rates of the coarser size fractions are often orders of magnitude smaller than those of the finer fractions, and the size distribution of the transported sediment has a smaller mean and variance than that of the bed [e.g., Jackson and Beschta, 1982; Carling, 1988; Church et al., 1991; Kuhnle, 1992; Wilcock and McArdell, 1993; Lisle, 1995]. As flow increases, the transported load coarsens and its size distribution approaches that of the bed [e.g., Parker and Klingeman, 1982; Wilcock, 1992]. Because discharge duration decreases with magnitude, flows producing finer-grained transport have longer duration and, in streams with widely sorted bed material, can produce much of the annual sediment load, which can be considerably finer-grained than the riverbed material [Leopold, 1992].

A decrease in transport rate with increasing grain size may be attributed to a smaller entrainment frequency for coarse grains or to size-dependent differences in the velocity or displacement length of moving grains. Visual observations of transport in flumes suggest that the latter two are unlikely to explain the observed differences in transport rate: larger grains, when in motion, appear to move rapidly and for great distances. This observation is supported by analysis of films of mixed-size sediment transport wherein the velocity of individual grain motions was found to be independent of grain size and the length of individual grain displacements was found to increase with grain size [Drake et al., 1988]. Thus the comparatively small transport rates observed for larger grains appears to result from a strong size dependence in entrainment rate.

Wilcock and McArdell [1993] suggested that the entrainment frequency of a size fraction depends not just on the rate at

Copyright 1997 by the American Geophysical Union.

Paper number 96WR02672.

0043-1397/97/96WR-02672\$09.00 which individual grains are entrained but also on the proportion of grains of a given size that are never entrained over the duration of a transporting event. For a given flow strength a size fraction may be composed of two populations: grains that move with some measurable regularity and grains that remain immobile, even though they are exposed on the bed surface. This condition, which they termed partial transport, was suggested by observing apparently immobile grains through the sidewalls of a flume. Partial transport may be defined for individual size fractions or for the bed as a whole. In the latter case, some proportion of the grains exposed on the bed surface, regardless of size, are transported while the remainder of the surface grains remain immobile.

Previous observations of a condition of partial transport have been made by Drake et al. [1988], who distinguished between active and inactive grain populations using detailed observations of grain motion on motion picture film. At the scale of an entire transport event the distinction between inactive grains (immobile over the entire transport event) and active grains (entrained into motion at least once) is readily made using tracer gravels. This distinction must be explicitly accounted for when calculating mean values of displacement length and grain velocity from tracer displacements [e.g., Church and Hassan, 1992; Hassan et al., 1992]. Stelczer [1981] used tracer gravels of size $D_{80}$ (the size for which $80 \%$ of the bed is finer) at three sites on the Danube River to measure the range of near-bottom velocity over which entrainment occurred. Stelczer [1981, p. 151] proposed that "to each particle size a lowest bottom velocity ... exists, below which there is no movement.... On the other hand, there exists also a bottom velocity at which all particles belonging to this fraction will move." Partial transport evidently prevails in the region between these two near-bed flow velocities. Stelczer [1981] also cited earlier work in the Russian literature in which a range of near-bed velocity for grain entrainment is specified. Wilcock et al. [1996a] also observed that entrainment of all surface grains 
within individual size fractions occurs over a range of flow strength.

Partial transport, if dependent on grain size, should evidently contribute to the observed decrease in transport rate with grain size. A demonstration of its existence and domain should increase our understanding of the dynamics of mixedsize sediment transport and improve our ability to develop representative models of the transport rate of individual size fractions. To provide a direct demonstration of partial transport, we used time series of bed photographs to measure the proportion of grains remaining immobile over the duration of flume runs of different flow strength. Here, we demonstrate the existence of partial transport; describe its variation with time, grain size, and flow strength; and illustrate its effect on fractional transport rates.

\section{Significance of Partial Transport}

The observation that only some of the particles in a given size range are mobile, if generally true, has important implications for modeling the entrainment and transport of mixedsize sediment and the exchange of sediment between the bed surface and subsurface.

The rate and size distribution of transported sediment depend not just on the population of grains on the bed surface [Parker, 1990], but on the proportion of those grains that can be mobilized by the flow, whether under conditions of zero sediment input leading to armoring [Proffitt and Sutherland, 1983] or with steady state transport composed of grains entrained from the bed upstream. Partial transport directly affects the rate and size of vertical sediment exchange between the bed surface and subsurface. If a large portion of the bed is immobile, the number of possible sites for vertical exchange will be limited. Further, if the active grains are primarily smaller ones, sediment exchange between bed surface and subsurface will be limited to the smaller grains that may pass through pockets vacated by entrained grains. A means of forecasting the active proportion of the bed surface is necessary for predicting any process that depends on grain sorting and sizeselective transport, including bed armoring, selective deposition, downstream fining, and the flushing or infiltration of fine-grained sediment into the subsurface of a gravel bed.

Theoretical models of mixed-size transport must account for the frequency of grain entrainment and either the velocity or displacement length of moving grains. The entrainment frequency may be expected to vary with grain size $D_{i}$ and bed shear stress $\tau_{0}$. If a portion of a fraction is immobile over the course of a transport event, the apparent entrainment frequency (e.g., the spatial entrainment rate which, when multiplied by mean grain displacement length, gives the transport rate) will differ from the actual entrainment frequency of only those grains that are actively transported. If a large proportion of a fraction is immobile, the apparent entrainment rate will be much smaller than the actual entrainment rate of active grains. Entrainment will be more accurately modeled if the proportion entrained and the entrainment rate of active grains are considered separately [Wilcock, this issue].

Partial transport is relevant to the definition of a characteristic exchange time for mixed-size beds, which is needed to represent the time required for a mixed-size bed to adjust to the prevailing flow and to define appropriate averaging times for observations of entrainment and transport, particularly for the coarser sizes on the bed surface. A useful time standard would be the time required to entrain a large proportion (e.g., 0.9 ) of the surface grains that are actively transported at a given flow. Under conditions of partial transport, active grains may be a small fraction of the total surface population, so that identification of the active proportion is necessary to accurately determine the exchange time.

The depth of sediment exchange within a mixed-size bed is relevant to models of size sorting in both the vertical and downstream directions. Exchange depth is commonly modeled as an active layer with a constant thickness equal to size of one of the coarser grains on the bed surface. Using the concept of partial transport, the spatial variability of the depth of grain exchange is shown to depend explicitly on the size distribution of active and inactive surface populations. A means of describing the degree of partial transport and its variation with grain size permits a more accurate representation of the exchange layer and a more realistic calculation of its thickness [Wilcock, this issue].

\section{Experimental Setup \\ Sediment}

The sediment used in the experiments varies in size from 0.21 to $64 \mathrm{~mm}$ ("bulk" in Figure 1), a size distribution that is coarser and more poorly sorted than normally used in flumes, but which is representative of many gravel-bed rivers. Another unusual feature of the sediment is that it was entirely separated into 14 size fractions and each fraction painted a different color. Standard $1 / 2 \phi$ size intervals were used for all fractions coarser than $1.0 \mathrm{~mm}$; the finest two fractions were $0.21-0.50$ $\mathrm{mm}$ and $0.50-1.0 \mathrm{~mm}$.

The primary motivation for coloring the sediment was to permit accurate and nonintrusive measurement of the bedsurface size distribution from photographs. Point counts were made by projecting photographic transparencies on a grid. Individual surface samples consisted of 980 points counted on 14 contiguous photographs covering a $40 \mathrm{~cm} \times 196 \mathrm{~cm}$ bed area. The large number of points was necessary to accurately estimate the surface proportion $F$, of each size fraction. Further detail regarding sediment preparation, sampling strategy, and accuracy of the surface sampling method are given by Wilcock and McArdell [1993].

\section{Flume Runs and Measurements}

The experiments were conducted in a tilting laboratory flume in which both water and sediment were recirculated. It has been noted previously that a condition of partial transport is produced most directly and with the fewest constraints using a sediment-recirculating flume [Parker and Wilcock, 1993; Wicock and McArdell, 1993]. The flume channel is $60 \mathrm{~cm}$ wide with a working length of $790 \mathrm{~cm}$. The entire transported load was sampled at the downstream end of the flume. The mean skin-friction shear stress was calculated using the mean velocity, water surface slope, and bed-surface size distribution in the Einstein [1950] drag-partition approximation. Further detail on the experimental procedure and the transport and hydraulic measurements are given by Wilcock and McArdell [1993].

Grain immobility observations were made for five runs covering a range in mean bed shear stress $\tau_{0}$ from 2.0 to $7.3 \mathrm{~Pa}$ and a range in transport rate $q_{b}$ from $7.5 \mathrm{~g} \mathrm{~m}^{-1} \mathrm{~s}^{-1}$ to $570 \mathrm{~g} \mathrm{~m}^{-1}$ $\mathrm{s}^{-1}$ (Table 1). These ranges include conditions of incipient motion for the coarse half of the sediment. Three of the runs were part of an earlier series made to provide coupled obser- 

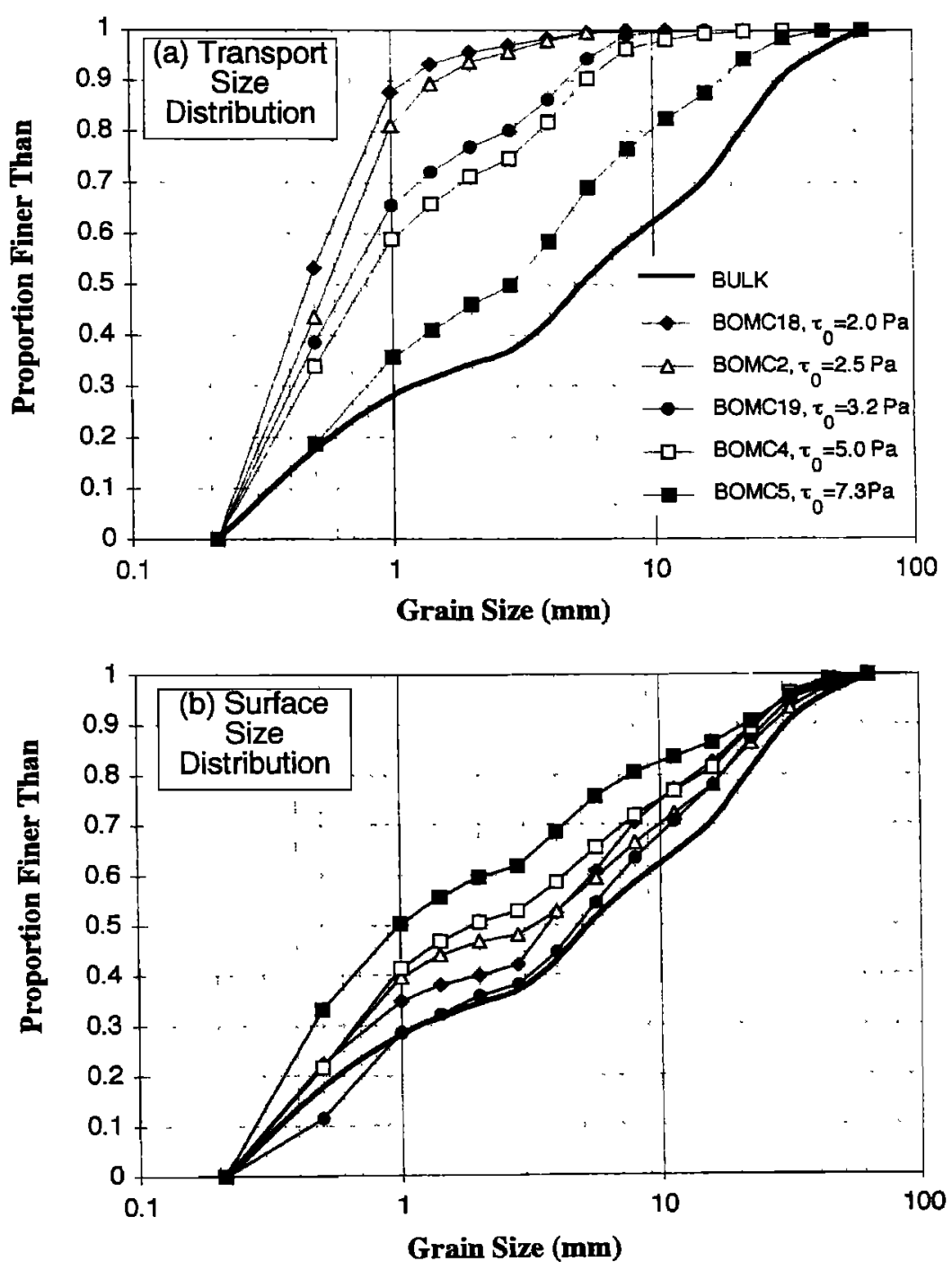

Figure 1. Grain-size distributions for the (a) transport and (b) bed surface during the experimental runs. The photographs used to measure the bed size distribution for BOMC18 and BOMC19 were selected to have a smaller proportion of fine fractions, so that the immobility of grains as small as $4.00 \mathrm{~mm}$ could be measured. The proportion of coarse fractions in transport increases with flow strength and is underrepresented relative to the bed surface except for BOMC5, the run with largest flow strength. The bed surface becomes finer with increasing flow strength, which is attributed to the release of subsurface fines by the increasing proportion of entrained coarse grains.

vations of flow, bed surface grain-size distribution, and fractional transport rates [Wilcock and McArdell, 1993]. Two additional runs were made to provide a longer duration and favorable photographic conditions for identifying grain immobility of size fractions as small as $D_{50}$ of the bulk mix.

\section{Bed Surface Texture and Transport Interactions}

As $\tau_{0}$ and $q_{b}$ increase, an increasing proportion of coarser grains become active, causing the size distribution of the transported grains to shift toward larger sizes (Figure 1a). In con-

Table 1. Mean of Hydraulic and Transport Observations for the Experimental Runs

\begin{tabular}{lccccccc}
\hline & $\begin{array}{c}\text { Flow } \\
\text { Depth, } \\
\text { cm }\end{array}$ & $\begin{array}{c}\text { Water } \\
\text { Surface } \\
\text { Slope, } \\
\%\end{array}$ & $\begin{array}{c}\text { Mean Flow } \\
\text { Velocity, } \\
\text { cm s s }^{-1}\end{array}$ & $\begin{array}{c}\text { Surface } \\
\text { Velocity, } \\
\mathrm{cm} \mathrm{s}^{-1}\end{array}$ & $\begin{array}{c}\text { Bed } \\
\text { Shear } \\
\text { Stress, } \\
\text { Pa }\end{array}$ & $\begin{array}{c}\text { Total } \\
\text { Transport } \\
\text { Rate, } \\
\mathbf{g ~ m}^{-1} \mathbf{s}^{-1}\end{array}$ & $\begin{array}{c}\text { Duration of Grain } \\
\text { Immobility } \\
\text { Observations, } \\
\text { Hours }\end{array}$ \\
\hline BOMC18 & 12.2 & 0.240 & 55 & 80 & 2.0 & 7.5 & 64.7 \\
BOMC2 & 11.2 & 0.315 & 60 & 80 & 2.5 & 7.1 & 27.9 \\
BOMC19 & 12.6 & 0.385 & 66 & 91 & 3.2 & 25.7 & 32.6 \\
BOMC4 & 9.4 & 0.765 & 87 & 111 & 5.0 & 157 & 9.2 \\
BOMC5 & 8.8 & 1.62 & 108 & 149 & 7.3 & 572 & 3.5 \\
\hline
\end{tabular}




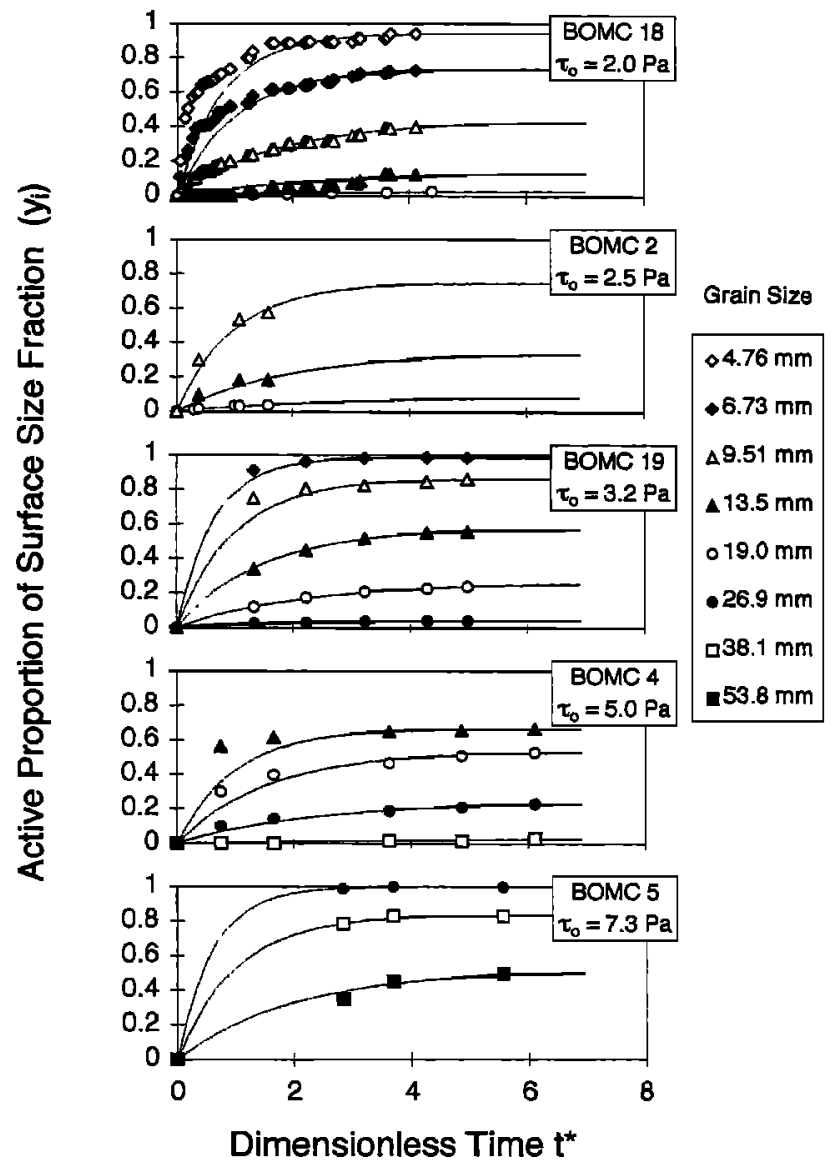

Figure 2. Proportion of surface grains of each size entrained over the course of a run, as a function of dimensionless time $t^{*}$. A grain is considered active if it is entrained at least once. The active fraction increases with time, approaching a constant value $Y_{i}$ at $2<t^{*}<6$. Curves are $y_{i}^{*}=\exp \left(-k t^{*}\right)$, with fitted values of $k$ and $Y_{i} ; y^{*}$ and $t^{*}$ defined in (1) and (2).

trast, the bed surface becomes progressively finer with increasing $\tau_{0}$ (Figure $1 \mathrm{~b}$ ). We attribute this to a progressive "mining" of finer sizes from the subsurface as the entrained proportion of coarse grains on the bed surface increases with $\tau_{0}$, supplying additional subsurface fine-grained sediment to the active transport within the closed flume system [Wilcock and McArdell, 1993].

The actively transported grains tend to collect in migrating grain-scale bed forms that are typically evident only as a somewhat finer-grained region of the bed; their thickness is typically less than the larger grains on the bed surface. Only during the run with the largest flow strength was the patch thickness sufficient to occasionally bury the larger immobile grains on the bed surface. Passage of the sandy patches was evident from slight changes in local bed elevation that altered the degree of burial of immobile grains. For the two new runs, BOMC18 and BOMC19, the timing of the bed photographs was chosen to minimize the proportion of sand in the photographed areas, which facilitated observations of the immobility of grains as small as $4.0 \mathrm{~mm}$. As a result, the surface size distributions for these runs have less sand than the earlier runs, so that the evolution with $\tau_{0}$ of the bed-surface size distribution does not follow the same pattern (Figure 1b).

\section{Grain Immobility Observations}

In each run the proportion of active grains was measured with respect to the bed surface population at an initial time shortly after the beginning of a run. Photographic slides of the initial population were projected on a grid on which the position of all grains of a given size were traced. Subsequent photographs were projected on the same drawings, and the presence or absence of particular grains was noted. A grain was classified to have been entrained if it moved entirely outside of its initial traced outline. Such small movement was observed only in rare cases; typically, entrained grains were no longer visible in the photographic frame. Distinctive wear patterns of the paint on individual grains and the arrangement of nearby immobile grains gave us a high degree of confidence that immobile grains were accurately identified. Accuracy in identifying entrained (as opposed to buried) grains was maintained by confirming the absence of a grain at a later time when the bed elevation was judged to be lower on the basis of relative exposure of neighboring immobile grains.

Most of the photographs were taken when the flume was drained. To provide further documentation of the mobility of the finest four fractions during $\mathrm{BOMC18}$, additional photographs were taken through a plastic sheet floating on the water surface while the run was in progress, taking care to avoid flow convergence and surface waves.

The active proportion of each size fraction $y_{i}$ increases rapidly at the start of the measurement period and approaches a limiting value $Y_{i}$ at large time (Figure 2). To facilitate comparison among different fractions and runs, both $y_{i}$ and timet were scaled. The scaled version of $y_{i}$,

$$
y_{i}^{*}=\left(Y_{i}-y_{i}\right) / Y_{i}
$$

varies between one at time $t=0$ and zero as $y_{i}$ approaches $Y_{i}$. Time was expressed as the total mass of transported sediment scaled by an approximate measure of the active mass of sediment in the flume:

$$
t^{*}=t q_{b} / \rho_{b} \lambda D_{a}
$$

where $\rho_{b}$ is bulk density of the bed, $\lambda$ is the length of bed, and $D_{a}$ is the smallest size that remains immobile over the course of the run. Equation (2) approximates the ratio of cumulative sediment transport to the mass in the active bed layer, providing an estimate of the number of times the active layer is cycled through the flume.

Values of $Y_{i}$ were found by regressing $\ln \left(y_{i}^{*}\right)$ on $t^{*}$ and adjusting $Y_{i}$ until the sum of squared deviations was minimized. This fit assumes an exponential decrease in $y_{i}^{*}$ of the form $y_{i}^{*}=\exp \left(-k t^{*}\right)$, where $k$ is the fitted slope and the intercept is equal to 1 at $t^{*}=0$ by the definition of $y_{r}^{*}$. The corresponding immobility observations and fitted $Y_{i}$ are given in Table 2. Because $y_{i}^{*}$ is specified at $t^{*}=0$ and $t^{*}=\infty$, values of $k$ are constrained to covary with $Y_{i}$. The fitted relations are shown in Figure 2, on which $y_{i}^{*}$ has been converted back to $y_{i}$ in order to separate the trends for individual size fractions. Other relations between $y_{2}^{*}$ and $t^{*}$ were tried, (e.g., power law) and similar values of $Y_{z}$ were produced.

In six cases the least squares fit was overridden and the value of $Y_{i}$ adjusted slightly to give a more credible fit to the data and to preserve similarity in form among the curves for each run. These are $D_{i}=19.0$ and $26.9 \mathrm{~mm}$ for BOMC18, for which very few grains moved, meaning that the variation of $\ln \left(y_{*}^{*}\right)$ with $t^{*}$ was dominated by a few large step changes in the value 
Table 2. Grain Immobility Observations

\begin{tabular}{|c|c|c|c|c|c|c|c|c|c|}
\hline \multirow{2}{*}{$\begin{array}{l}\text { Duration of } \\
\text { Immobility } \\
\text { Observations } t^{*}\end{array}$} & & \multicolumn{8}{|c|}{ Grain Immobility Observations for Individual Size Fractions, mm } \\
\hline & & 53.8 & 38.1 & 26.9 & 19.0 & 13.5 & 9.51 & 6.73 & 4.76 \\
\hline \multicolumn{10}{|c|}{ BOMC18 } \\
\hline 4.1 & $\begin{array}{l}N_{\text {init }} \\
N_{\text {imm }} \\
Y_{t}\end{array}$ & $\begin{array}{c}15 \\
15 \\
0.00\end{array}$ & $\begin{array}{c}47 \\
47 \\
0.00\end{array}$ & $\begin{array}{l}200 \\
199 \\
0.01\end{array}$ & $\begin{array}{c}433 \\
422 \\
0.04^{a}\end{array}$ & $\begin{array}{c}80 \\
70 \\
0.15^{a}\end{array}$ & $\begin{array}{r}125 \\
75 \\
0.44\end{array}$ & $\begin{array}{r}114 \\
31 \\
0.74\end{array}$ & $\begin{array}{r}105 \\
6 \\
0.95\end{array}$ \\
\hline 1.6 & $\begin{array}{l}N_{\text {init }} \\
N_{\text {Imm }} \\
Y_{1}\end{array}$ & $\begin{array}{c}19 \\
19 \\
0.00\end{array}$ & $\begin{array}{c}30 \\
30 \\
0.00\end{array}$ & $\begin{array}{l}D M C 2 \\
106 \\
105 \\
0.01\end{array}$ & $\begin{array}{c}196 \\
189 \\
0.10^{\alpha}\end{array}$ & $\begin{array}{c}116 \\
95 \\
0.35^{a}\end{array}$ & $\begin{array}{r}124 \\
52 \\
0.75^{a}\end{array}$ & $\begin{array}{c}\cdots \\
\cdots \\
0.95^{b}\end{array}$ & $\begin{array}{c}\ldots \\
0.99^{b}\end{array}$ \\
\hline 5.0 & $\begin{array}{l}N_{\text {init }} \\
N_{1 \mathrm{~mm}} \\
Y_{t}\end{array}$ & $\begin{array}{c}20 \\
20 \\
0.00\end{array}$ & $\begin{array}{c}53 \\
53 \\
0.00\end{array}$ & $\begin{array}{l}M C 19 \\
213 \\
204 \\
0.05\end{array}$ & $\begin{array}{l}446 \\
339 \\
0.27\end{array}$ & $\begin{array}{l}236 \\
104 \\
0.58\end{array}$ & $\begin{array}{r}291 \\
40 \\
0.87\end{array}$ & $\begin{array}{r}357 \\
5 \\
0.99\end{array}$ & $\begin{array}{l}\cdots \\
1.00\end{array}$ \\
\hline 6.1 & $\begin{array}{l}N_{\text {inut }} \\
N_{\text {imm }} \\
Y_{\imath}\end{array}$ & $\begin{array}{c}10 \\
10 \\
0.00\end{array}$ & $\begin{array}{c}64 \\
62 \\
0.10\end{array}$ & $\begin{array}{c}O M C 4 \\
190 \\
146 \\
0.25\end{array}$ & $\begin{array}{l}307 \\
144 \\
0.54\end{array}$ & $\begin{array}{r}117 \\
39 \\
0.67\end{array}$ & $\begin{array}{c}\cdots \\
\cdots \\
1.00^{b}\end{array}$ & $\begin{array}{l}\cdots \\
1.00\end{array}$ & $\begin{array}{l}\cdots \\
1.00\end{array}$ \\
\hline 5.6 & $\begin{array}{l}N_{\text {init }} \\
N_{\text {imm }} \\
Y_{1}\end{array}$ & $\begin{array}{c}20 \\
10 \\
0.52^{a}\end{array}$ & $\begin{array}{c}65 \\
11 \\
0.84\end{array}$ & $\begin{array}{c}O M C 5 \\
98 \\
0 \\
1.00\end{array}$ & $\begin{array}{c}\ldots . \\
\ldots \\
1.00\end{array}$ & $\begin{array}{c}\ldots \\
\ldots \\
1.00\end{array}$ & $\begin{array}{l}\ldots \\
\cdots \\
1.00\end{array}$ & $\begin{array}{c}\ldots \\
\ldots \\
1.00\end{array}$ & $\begin{array}{c}\ldots \\
\ldots \\
1.00\end{array}$ \\
\hline
\end{tabular}

$N_{\text {init }}$ initial number observed; $N_{\text {imm }}$, final number remaining immobile; $Y_{i}$, estimated proportion of active grains at large $t^{*}$.

${ }^{a} Y_{i}$ fitted by eye.

${ }^{b}$ No immobility observations; assumed value of $Y_{i}$.

of $y_{i}^{*} ; D_{i}=9.51,13.5$, and $19.0 \mathrm{~mm}$ for BOMC2, which was a relatively short run, so that $y_{i}$ had apparently not yet approached $Y_{i}$; and $D_{i}=53.8 \mathrm{~mm}$ for BOMC5, which had only three observations, providing a less certain fit. Less significance is assigned to these points, particularly those from BOMC2 because the run is relatively short. In each case, the curves shown in Figure 2 use the values of $Y_{1}$ we carry forward in the analysis.

Although $y_{i}$ for all fractions show an asymptotic approach to $Y_{i}, y_{i}$ for the finer fractions tends to increase more rapidly at small $t^{*}$. This suggests that most of the active proportion of the finer fractions is relatively easily entrained. Displacement of the remaining active fine grains may require entrainment of the larger grains, which proceeds at a slower rate.

Because of flow turbulence, $Y_{i}$ is not an absolute maximum (runs of sufficient length would presumably entrain additional grains), but the rate at which additional grains are entrained becomes negligible and $y_{i}$ becomes essentially constant for runs of practical duration. Rather than pursue the general case $y_{i}=y_{i}\left(D_{i}, \tau_{0}, t\right)$, we consider here the steady state case $Y_{i}=$ $Y_{i}\left(D_{2}, \tau_{0}\right)$, focusing on the quasi steady state portion of the curve for which further change in $y_{i}$ is negligible.

This raises the question of whether a characteristic run duration exists over which most of the mobilizable grains will be entrained. Much of the change in $y_{i}$ has occurred once $t^{*}$ exceeds a value of approximately two (Figure 2). At $t^{*}=2$ the mean value of $y_{i}^{*}$ is 0.24 for all measured cases. At $t^{*}=4$ the mean of all $y_{i}^{*}$ is 0.08 and $y_{i}^{*}<0.2$ for all but one case. At $t^{*}=$ 6 mean $y_{i}^{*}$ is 0.03 and $y_{i}^{*}<0.1$ for all but one case. This suggests that $t^{*}$ can be used to provide a useful grain exchange time for conditions of partial transport. First, the active mass of sediment on the bed surface is defined using $Y_{i}$ and the proportion $F_{i}$ of each fraction on the bed surface. The time nec- essary to entrain, say, $90 \%$ of that mass may then be estimated as that necessary to produce a cumulative transported mass approximately 4 times that of the active mass. Such an exchange time provides a physical basis for specifying a sampling or averaging interval for the entrainment or transport of coarser size fractions under conditions of partial transport.

A further point regarding adjustment time is worth noting. At small transport rates the actual time required to reach steady state may be quite long. For BOMC18, more than 60 hours is required to reach $t^{*}=4$. This suggests that under natural conditions with variable discharge, the local streambed may often not be in equilibrium with the prevailing flow.

\section{Variation of Grain Mobility With Grain Size and Bed Shear Stress}

The variation of $Y_{i}$ with $\tau_{0}$ and $D_{i}$ demonstrates the existence and nature of partial transport (Figure 3a). The entrainment of grains from a particular surface fraction does not occur at a single $\tau_{0}$ but over a range of $\tau_{0}$, so that the transition from immobility $\left(Y_{i}=0\right)$ to full mobility $\left(Y_{t}=1\right)$ occurs over a range of shear stress. For a given shear stress the same transition occurs over a range in grain size.

Although the data are limited in number and some scatter is evident, an approximating distribution for $Y_{i}\left(\tau_{0}\right)$ provides a useful device for describing the domain of partial transport. A lognormal distribution $Y_{i}\left(\tau_{0}\right)$, shown in cumulative form in Figure $3 \mathrm{~b}$, provides a simple distribution that is restricted to positive values of $\tau_{0}$ and gives a plausible fit to the data. The choice of a lognormal distribution of $\tau_{0}$ is supported by the observation that the distribution of pivot angles for the entrainment of individual size fractions in a mixed-size sediment 

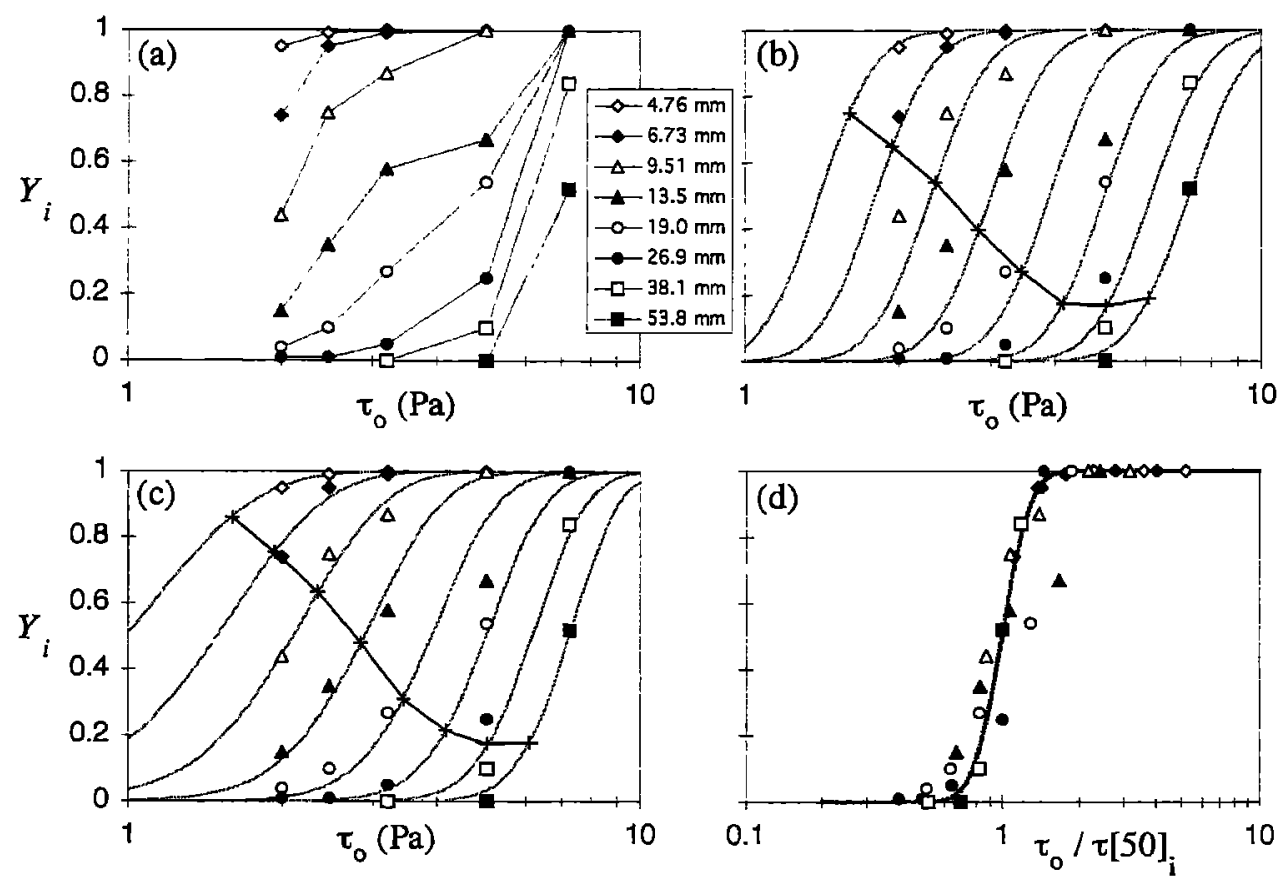

Figure 3. Active proportion $Y$, for each fraction as a function of grain size and bed shear stress. The same data symbols are used in each panel. (a) $Y_{i}$ as a function of $\tau_{0}$. (b) Curves are lognormal cumulative distributions with standard deviation $\sigma=0.2$ and mean $\tau[50]_{i}$ fitted by eye to each fraction. (c) Curves are normal cumulative distributions of $u_{f}$ using $\sigma=0.06 \mathrm{~m} \mathrm{~s}^{-1}$, (3) to convert $u_{f}$ to $\tau_{0}$, and a mean $\tau[50]_{i}$ fitted by eye to each fraction. (d) $Y_{i}$ as a function of $\tau_{0} / \tau[50]_{i}$, using $\tau[50]_{i}$ values from Figure $3 \mathrm{~b}$. The intersecting lines in Figures $3 \mathrm{~b}$ and $3 \mathrm{c}$ (marked with pluses) are the value of $Y_{t}$ at the reference transport rate $\tau_{r i}$ for each fraction. For a lognormal distribution of $\tau_{0} / \tau[50]_{i}$, the transition $0.1<Y_{i}<0.9$ occurs over a range of $\tau_{0}$ of a factor of 2 and over a range of $D_{i}$ of a factor of 2 . Values of $Y_{i}$ at $\tau_{0}=5 \mathrm{~Pa}$ (BOMC4) appear to be small relative to the remainder of the data and were given less weight in fitting the probability distributions.

follows a lognormal form [Johnston, 1996]. The distributions plotted use a constant standard deviation $\sigma=0.2$ for all sizes and a mean shear stress $\tau[50]_{i}$ (for which $Y_{i}=0.5$ ) fitted by eye. The sparse data and its scatter preclude a rigorous fit for each size, so both curves and data are shown in Figure $3 \mathrm{~b}$. Values of $Y_{i}$ at $\tau_{0}=5 \mathrm{~Pa}$ (run BOMC4) appear to be small relative to the values of adjoining runs and were given less weight in fitting the distributions. For a standard deviation of 0.2 the transition $0.1<Y_{i}<0.9$ occurs over a range of $\tau_{0}$ of a factor of 2; for the spacing of the cumulative curves in Figure $3 \mathrm{~b}$ the transition $0.1<Y_{i}<0.9$ occurs over a range in grain size of approximately a factor of 2 .

Stelczer [1981, p. 162] found similar results using the active proportion of surface tracer gravels along three cross sections of the Danube River. The tracer grain sizes were 14.2, 25.8, and $31.4 \mathrm{~mm}$, which correspond to $D_{80}$ at the three sites. Stelczer found that the range of conditions over which entrainment occurred could be represented using a normal distribution of "bottom velocity" $u_{f}$, with a standard deviation of $0.06 \mathrm{~m} \mathrm{~s}^{-1}$ in each case. In this case the transition $0.1<Y_{i}<$ 0.9 occurs over a range of $u_{f}=0.15 \mathrm{~m} \mathrm{~s}^{-1}$. An approximate comparison with the lognormal $\tau_{0}$ distribution in Figure $3 \mathrm{~b}$ can be made using a standard logarithmic velocity profile. Stelczer calculated $u_{f}$ as one half the velocity at a height of $0.3 \mathrm{~m}$ above the bed, so an appropriate form is

$$
u_{f}=\frac{1}{2}\left[2.5\left(\tau_{0} / \rho\right)^{1 / 2} \ln \left(\frac{30 z}{k_{s}}\right)\right]
$$

where $k_{s} / 30$ is the Nikuradse value of $z$ at which $u_{f}$ goes to zero, and the roughness $k_{s}$ is taken to be $D_{80}$. For consistency $z=0.3 \mathrm{~m}$, even though depths this large were not used in the experimental runs.

The fitted distributions for a normal $u_{f}$ distribution with $\sigma=$ $0.06 \mathrm{~m} \mathrm{~s}^{-1}$ are given in Figure 3c, in which the mean value for each fraction is again fitted by eye. The distribution is similar to that of Figure $3 b$ for the coarse fractions; the standard deviation for the normal $u_{f}$ distribution becomes larger for the finer fractions, providing an apparently better fit for the 9.51-, $13.5-$, and $19.0-\mathrm{mm}$ fractions. The scatter in the data, the absence of $Y$, observations for the finest fractions, and the similar fit for the coarsest fractions prevent a clear choice between the two distributions.

Although we cannot definitively specify a particular distribution of $\tau_{0}$ or $u_{f}$ to represent fractional entrainment, it is clear that fractional entrainment occurs over a range of flow strength and that this range increases consistently with grain size. Of most interest in investigating the relative mobility and transport of different sizes is the relative position of the fitted distribution for each size fraction. The range of $\tau[50]_{i}$ that provides a plausible fit to the data in Figure $3 b$ and $3 c$ is relatively limited, and may be further constrained by arguing that $\tau[50]_{i}$ should vary smoothly with increasing $D_{i}$. A collapse of the data using $\tau_{0} / \tau[50]_{i}$ (Figure 3d), where $\tau[50]_{i}$ is the mean of the lognormal $\tau_{0}$ distributions in Figure $3 \mathrm{~b}$, suggests that a constant- $\sigma$ lognormal $\tau_{0}$ distribution provides sufficient accuracy to illustrate the domain of partial transport and may be used to approximate the variation of fractional entrainment with $\tau_{0}$. Although we consider further both $\tau_{0}$ and 


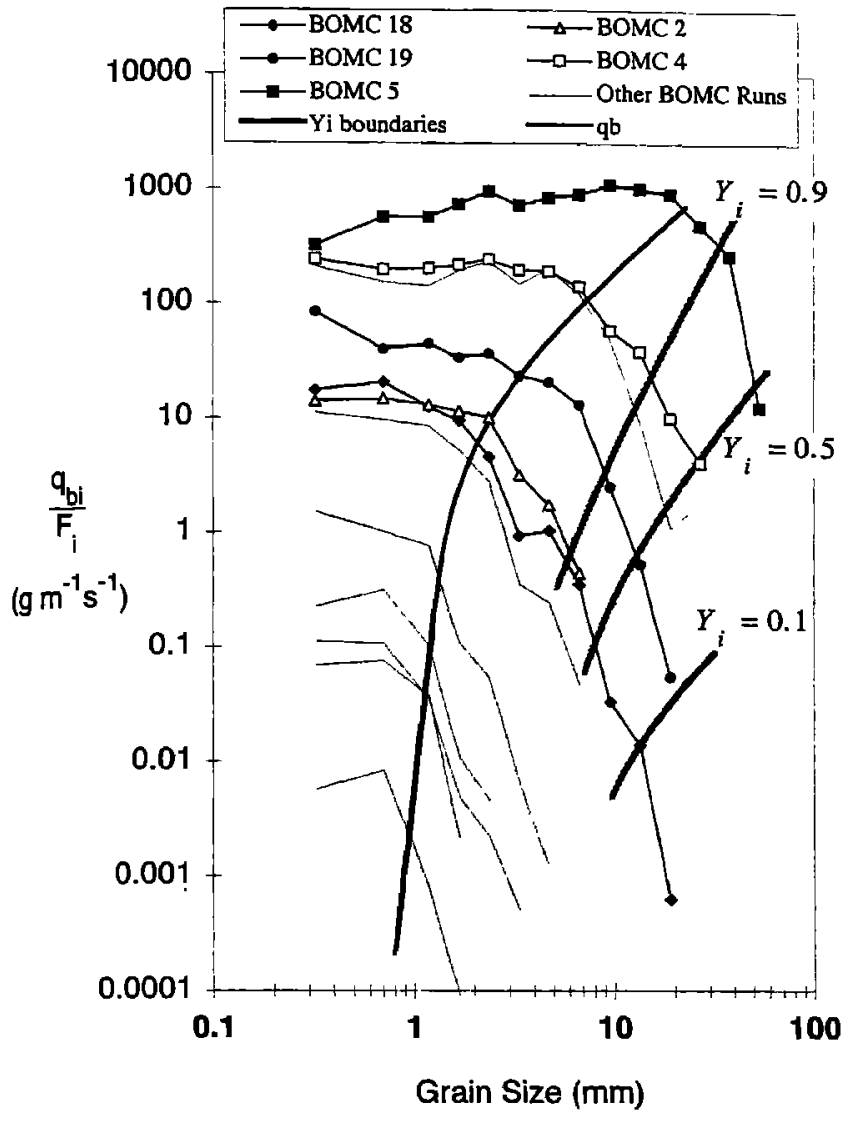

Figure 4. Fractional transport rates as a function of grain size, with contours of $Y_{i}$ superimposed to represent the degree of partial transport for each run. Contours of $Y_{i}=0.1,0.5$, and 0.9 determined by interpolation on Figure $3 \mathrm{~b}$ to find the associated value of $D_{i}$ for $\tau_{0}$ of each run. The total transport rate $q_{b}$ is plotted where it intersects the fractional transport trends for each run, approximating the size for which $p_{i}=F_{i}$. Equal mobility appears as approximately size-independent trends in $q_{b_{l}} / F_{i}$ to the left of the $q_{b}$ curve; a rapid decrease in $q_{b i} / F_{i}$ exists for larger sizes, which are in a state of partial transport. Fully mobile $\left(Y_{i}=1\right)$ transport occurs within approximately 1 order of magnitude in $q_{b i} / F_{i}$ of the transition to equal mobility. $Y_{i}<0.1$ is associated with undetectably small transport for sizes coarser than $23 \mathrm{~mm}$.

$u_{f}$ distributions, more emphasis is placed on the $\tau_{0}$ distribution, which permits more direct comparison with existing transport relations [Wilcock, this issue] and does not require independent specification of flow depth.

\section{Partial Transport and Fractional Transport Rates}

Fractional transport rates for the experimental runs are plotted as a function of $D_{i}$ in Figure 4. For completeness, transport rates for other BOMC runs are shown as light lines with no symbols. Transport is plotted as $q_{b i} / F_{i}$ where $q_{b i}=p_{i} q_{b} ; p_{i}$ and $F_{i}$ are the proportion of fraction $i$ in transport and on the bed surface, respectively; and $q_{b}$ is the mass transport rate per unit width. For the finer fractions in each run, $q_{b i} / F_{i}$ is essentially independent of $D_{i}$, whereas $q_{b i} / F_{i}$ decreases rapidly for larger sizes. The smaller fractions are in a state of equally mobile transport, defined as equivalent transport and bed- surface size distributions [Wilcock and McArdell, 1993]. For these fractions, $p_{1}=\alpha F_{1}$, where $\alpha$ is the active proportion of the bed surface and accounts for the fact that only part of the bed surface participates in the transport. In the limit of equal mobility for all fractions, $\alpha=1$ and $p_{i}=F_{i}$, as originally defined by Parker et al. [1982]. For a given run, $\alpha$ is a constant. so $q_{b j} / F_{z}=\alpha q_{b}$ is a constant for all equally mobile fractions.

For all runs, $q_{b i} / F_{2}$ decreases rapidly for grain sizes larger than a threshold that increases with flow strength from less than $1.0 \mathrm{~mm}$ to more than $20 \mathrm{~mm}$. The threshold is approximated by the curve for total transport rate $q_{b}$, which is plotted on Figure 4 at the intersection of $q_{b}$ and the $q_{b} / F_{i}$ trend for each run. This gives the grain size for which $p_{i} / F_{i} \approx 1$ in each run.

A direct comparison between partial transport and fractional transport rates is made by superimposing contours of $Y_{i}$ on Figure 4. To determine the $Y_{i}$ contours, the grain size associated with $Y_{i}=0.1,0.5$, and 0.9 for each run was determined using the associated value of $\tau_{0}$ and interpolating between the cumulative curves on Figure $3 \mathrm{~b}$. Values of $Y_{2}=$ 0.1 are associated with transport rates that are orders of magnitude smaller than those of fully mobile fractions. At $Y_{i}=$ 0.9 , fractions are approaching complete mobilization and the fractional transport rates fall within approximately 1 order of magnitude of the equally mobile fractions. There is some divergence between the curves for $q_{b}$ and $Y_{i}=0.9$ for smaller $D_{\imath}$. This may result from mobilized finer grains becoming trapped among immobile coarse grains over the duration of a transport event, thereby decreasing their proportion in the measured transport rate. This process would be more prevalent at smaller $\tau_{0}$, for which a larger proportion of the bed surface is immobile.

A comparison of partial transport and incipient motion conditions is given in Figure 5a. Incipient motion is approximated by the reference shear stress $\tau_{r i}$ that produces a small, reference transport rate of each size, which provides a well-defined and repeatable surrogate for the critical shear stress for incipient motion [Parker et al., 1982; Wilcock, 1988, 1993]. The reference transport rate used was $W_{i}^{*}=0.002$, where

$$
W_{i}^{*}=\frac{(s-1) g q_{b r}}{F_{t} \rho_{s} u_{*}^{3}}
$$

$\rho_{s}$ is the sediment density, $s$ is the relative sediment density $\rho_{s} / \rho, g$ is the acceleration of gravity, and $u_{*}$ is the shear velocity, given by $\left(\tau_{0} / \rho\right)^{1 / 2}$. Values of $\tau_{r t}$ for the BOMC sediment were determined by Wilcock and McArdell [1993]. For $D_{\imath}>2.8 \mathrm{~mm}, \tau_{r i}$ can be approximated by $\tau_{r l}=0.68 D_{\imath}^{0.55}$ for $\tau_{r i}$ in pascals and $D_{1}$ in millimeters. Also shown on Figure 5a is the value of $\tau_{0}$ producing $p_{i}=F_{i}$ (equal mobility) for each size, determined from plots of $p_{i} / F_{i}$ against $\tau_{0}$ [Wilcock and McArdell, 1993]. The space between this curve and that for $\tau_{r_{1}}$ was initially suggested by Wilcock and McArdell [1993] as the region of partial transport.

The shear stresses $\tau[10]_{i}, \tau[50]_{l}$, and $\tau[90]_{l}$ associated with $Y_{i}=0.1,0.5$, and 0.9 , respectively, were determined for each grain size from the $Y_{i}\left(\tau_{0}\right)$ distributions in Figure $3 \mathrm{~b}$. The entrainment and reference transport curves in Figure $5 \mathrm{a}$ illustrate the relation between partial transport and incipient motion; $\tau_{r l}$ falls between $\tau[90]_{i}$ and $\tau[50]_{l}$ for smaller sizes $\left(D_{t}<\right.$ $14 \mathrm{~mm}$ ) and decreases to smaller entrainment rates for the larger sizes, approaching the $\tau[10]_{i}$ curve for $D_{i}>19 \mathrm{~mm}$. The value of $Y_{i}$ associated with $\tau_{r i}$ for each fraction is shown directly on Figure $3 \mathrm{~b}$ and decreases with grain size from $Y_{l}=$ 

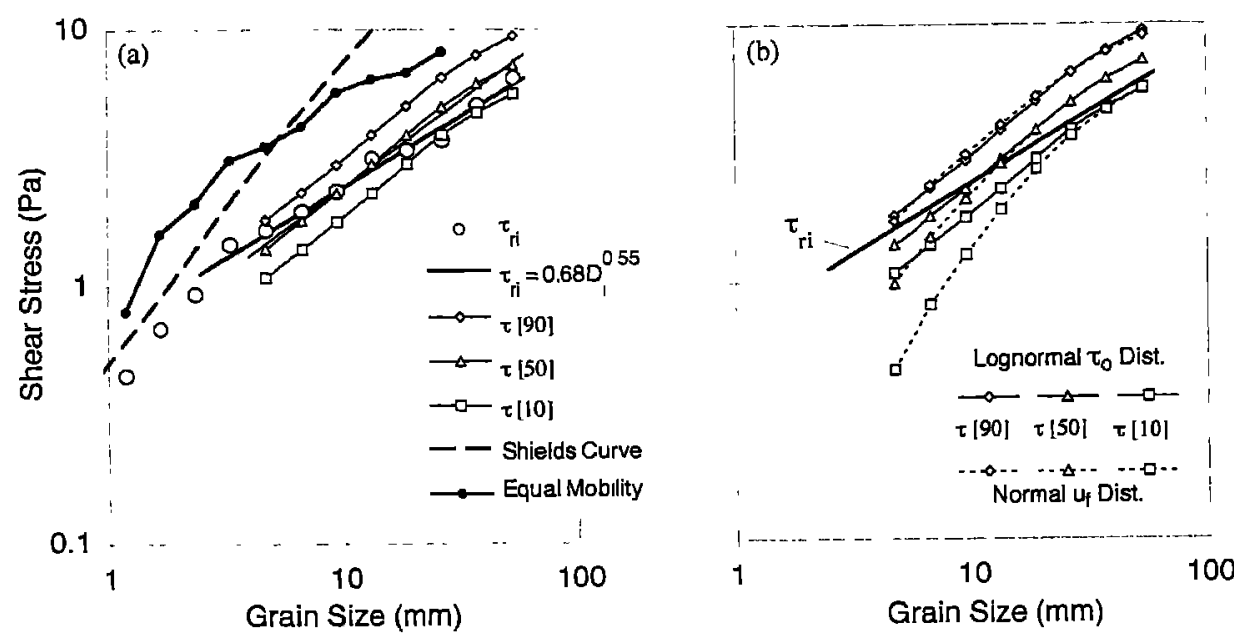

Figure 5. (a) Shear stress for various measures of grain entrainment, as a function of grain size. $\tau_{r i}$ is the reference shear stress, which can be approximated as $\tau_{r i}=0.68 D_{\imath}^{0.55}$ for sizes coarser than $2.8 \mathrm{~mm}$ [Wilcock and $M c$ Ardell, 1993]; $\tau[10]_{i}, \tau[50]_{i}$, and $\tau[90]_{i}$ are the shear stresses producing $Y_{i}=0.1,0.5$, and 0.9 for each size fraction, determined from the cumulative distributions in Figure $3 \mathrm{~b}$. The equal mobility threshold was determined as the value of $\tau_{0}$ giving $p_{i}=F_{i}$ on plots of $p_{i} / F_{i}$ versus $\tau_{0}$ [Wilcock and McArdell, 1993]. The Shields curve for incipient motion of unisize sediment is shown for comparison; $\tau_{r i}$ is associated with more than $50 \%$ bed entrainment $\left(Y_{1}>0.5\right)$ for the smaller sizes and $Y_{i} \approx 0.2$ for the coarsest sizes. (b) Comparison of $\tau[90]_{i}, \tau[50]_{i}$, and $\tau[10]_{i}$ from Figure 5a with corresponding values determined using a normal distribution in $u_{f}\left(\sigma=0.06 \mathrm{~m} \mathrm{~s}^{-1}\right.$; Figure $\left.3 \mathrm{c}\right)$.

0.75 to $Y_{i}=0.2$. A general case can be made that the mobilized proportion of a fraction decreases with grain size at the reference transport condition and that $\tau[50]$, may be predicted as a function of $\tau_{r l}$ [Wilcock, this issue]. Because $Y_{i}$ must be less than 1 , a decrease in $Y_{1}$ with grain size implies that partial transport is characteristic of reference transport conditions.

The separation between the equal mobility and $\tau[90]$, curves on Figure 5a decreases with grain size from roughly a factor of 2 at $D_{i}=4.8 \mathrm{~mm}$ to less than a factor of 1.3 at $D_{t}=$ $26.9 \mathrm{~mm}$. This corresponds to the similar trend between $q_{b}$ and $Y_{1}=0.9$ on Figure 4 and suggests that equal mobility $\left(p_{i}=F_{i}\right)$ coincides with full mobility $\left(Y_{t}=1\right)$ for larger, but not smaller, sizes.

The variation of $\tau[50]_{i}$ with grain size may be represented by the loglinear relation

$$
\tau[50]_{l}=0.523 D_{t}^{0.67}
$$

for $\tau_{0}$ in pascals and $D_{i}$ in millimeters (Figure 5a). The inverse of (5) gives the grain size $D[50]_{i}$ that is $50 \%$ mobilized at a given $\tau_{0}$. Because the range of sizes in the partial transport range is limited, $D[50]_{i}$ provides a convenient approximation for the depth of grain exchange $d_{x}$. A suitable dimensionless form is

$$
d_{\mathrm{\lambda}} / D_{50}=397\left(\tau_{50}^{*}\right)^{15}
$$

where $d_{x}=D[50]_{i}, \tau_{50}^{*}$ is the dimensionless shear stress $\tau_{0}\left[(s-1) \rho g D_{50}\right]^{-1}$, and the values $(s-1)=1.65, \rho g=$ $9800 \mathrm{~kg} \mathrm{~m}^{-2} \mathrm{~s}^{-2}$, and $D_{50}=0.0053 \mathrm{~m}$ have been used.

Values of $\tau[10]_{i}, \tau[50]_{i}$, and $\tau[90]_{i}$ obtained using the constant- $\sigma$ normal distribution of $u_{f}$ (Figure $3 \mathrm{c}$ ) are compared to those from the constant- $\sigma$ lognormal distribution of $\tau_{0}$ (Figure 3b) in Figure 5b. Values of $\tau[90]_{i}$ are similar for both distributions, as are values of $\tau[50]_{i}$ and $\tau[10]$, for the coarser

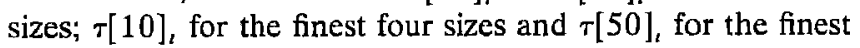

two sizes deviate between the two estimates, but these correspond to values of $Y_{i}$ for which no direct observations are available, so that $\tau[50]_{i}$ and $\tau[10]_{i}$ are speculative in this range. The relation between $\tau_{r i}$ and the degree of partial transport is similar for both distributions (Figure 5b). Both distributions support the general conclusions that mobilization of a size fraction occurs over a range of flow strength and that larger grains are mobilized at larger flows.

\section{Implications for Sediment Exchange Between Bed and Transport}

Partial transport determines the active proportion of the bed surface and therefore plays a direct role in controlling both the rate and size of sediment exchange between grains in motion, the bed surface, and the bed subsurface. The rate of infiltration or flushing of fine sediments in the bed subsurface, which is important to the quality of the substrate as habitat for fish and invertebrates, depends on the total proportion of the bed surface mobilized during a transport event. The active proportion of the bed surface during the BOMC runs is shown in Figure 6a. The active proportion is calculated relative to the size distribution of the entire mix as well as to the gravel portion of the bed surface. The latter is likely to provide a better indication of the degree of bed mobilization giving rise to vertical sediment exchange.

A substantial portion of the bed surface can remain immobile in the presence of nonnegligible transport rates. For example, at $\tau_{0}=3.2 \mathrm{~Pa}$ (BOMC 19) the total transport rate is $26 \mathrm{~g} \mathrm{~m}^{-1} \mathrm{~s}^{-1}$ and the gravel transport rate is $10 \mathrm{~g} \mathrm{~m}^{-1} \mathrm{~s}^{-1}$ (Figure 6c), yet more than one third of the gravel grains on the bed surface remain immobile over the run (Figure 6a). This transport rate is more than an order of magnitude smaller than the largest rate we measured and much larger transport rates occur during floods on gravel-bed rivers. However, the in- 

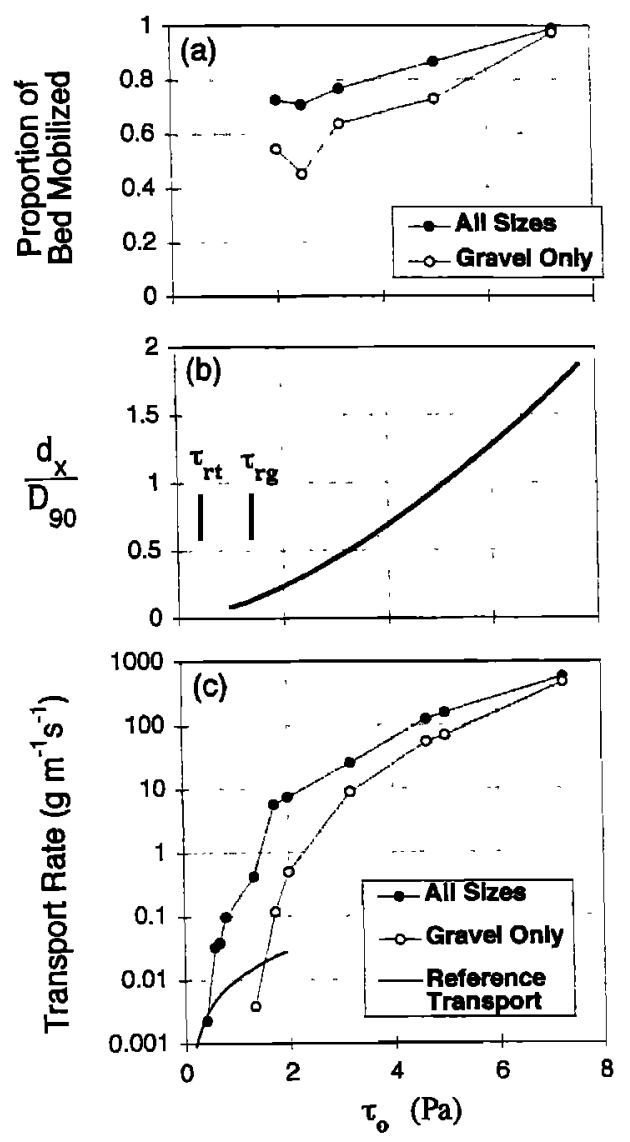

Figure 6. Total surface entrainment as a function of $\tau_{0}$. (a) Proportion of bed surface mobilized, calculated as $\Sigma\left(Y_{i} F_{i}\right)$, for all grain sizes and renormalized for the gravel portion of the bed. (b) Exchange depth $d_{x}$ (estimated as the grain size for which $Y_{i}=0.5$ ), scaled by $D_{90}=31 \mathrm{~mm} ; \tau_{r i}$ and $\tau_{r g}$ are the reference shear stresses for the total transport rate and for transport of the combined gravel fractions, respectively. (c) Total transport rates and transport rates for the gravel fraction (scaled by the gravel proportion of the bed surface). Mobilization of the gravel framework occurs over $\tau_{r g}<\tau_{0}<4 \tau_{r g}$. Substantial transport rates are associated with entrainment of less than two thirds of the bed surface (BOMC19; $\tau_{0}=3.2 \mathrm{~Pa}$ ); complete surface mobilization is associated with nearly equally mobile transport of the sand and gravel (BOMC5; $\tau_{0}=7.3 \mathrm{~Pa}$ ).

crease in discharge duration with decreasing magnitude can be sufficient that a substantial amount of the annual sediment transport occurs under these low transport conditions, particularly for steep rivers with widely sorted bed material and a dominant snow melt runoff regime [Leopold, 1992]. The prevalence of partial transport conditions is likely to be even greater in regulated rivers and during periods of drought.

Nearly the entire bed surface is mobilized at the largest transport rate we measured (Figure 6a). For this run (BOMC5), only the coarsest fraction (comprising less than $4 \%$ of the sediment mix) has values of $Y_{1}$ and $q_{b i} / F_{i}$ substantially smaller than the remaining fractions (Figure 4). At this flow the transport rate of the gravel portion of the bed is nearly equal to the total transport rate (Figure 6c), suggesting that complete mobilization of the bed surface (in the sense that all grains are entrained at least once) coincides with nearly complete equal mobility. This empirical result is not inevitable. A possible alternative, which we do not observe, is that equally mobile transport of the gravel would be produced by only a fraction of the surface grains under conditions of partial transport.

The coincidence of complete bed mobilization and equally mobile transport has important implications for the specification of flushing flows designed to mobilize the bed surface and remove fine sediments from the subsurface. One possible objective for an efficient flushing flow is to entrain all grains on the bed surface, thereby permitting flow access to the subsurface [Wilcock et al., 1996b]. For the BOMC sediment, we observe that transport rates must be increased to the point of near-equal mobility of all sizes in order to entrain all grains on the bed surface at least once. If all fractions are transported at the same rate (when scaled by their presence in the bed), the future composition of the bed will immediately and exactly equal that of the upstream sediment supply, which can be finer grained than the existing bed in channels below reservoirs. This suggests that the range of flows producing complete bed mobilization while maintaining selective transport of the finer fractions may be vanishingly small [Wilcock, 1995; Kondolf and Wilcock, 1996].

The thickness of the active layer, or depth of sediment exchange, is needed for models of vertical grain exchange, selective deposition, and armoring [e.g., Hirano, 1971; Proffitt and Sutherland, 1983; Parker and Sutherland, 1990; Parker, 1991]. It is also part of the calculation of subsurface flushing or infiltration. Equation (6) may be used to estimate the exchange depth $d_{x}$ as the size fraction $D[50]_{i}$ that is $50 \%$ mobile for a given flow (Figure 6b). At $\tau_{r g}=1.4 \mathrm{~Pa}$, which is the reference shear stress for the combined gravel fraction (at which nearly all of the gravel transport is composed of grains finer than $4.0 \mathrm{~mm}$ ), extrapolation on Figure 6 suggests that more than half of the bed surface is immobile and $d_{x}$ is only a small fraction of $D_{90}$. As $q_{b}$ increases, $d_{x}$ increases in proportion to $\tau_{0}^{1.5}$, reaching $d_{x} / D_{90}=1$ at $\tau_{0} \approx 5.0 \mathrm{~Pa}$, or 3 to 4 times $\tau_{r g}$. An exchange depth of $d_{x} \approx 2 D_{90}$ is associated with complete mobilization of the bed surface. The variation with $\tau_{0}$ of $D[50]_{i}$ (and $d_{x}$ ) can be shown to depend on the distribution of $\tau_{r i}$ [Wilcock, this issue] and should therefore vary from sediment to sediment. In particular, a sediment with a narrower range of $\tau_{r i}$ than the BOMC sediment will have a correspondingly more rapid increase of $d_{x}$ with $\tau_{0}$. We cannot state whether the limiting value of $d_{x}$ at full mobilization varies strongly with sediment size distribution, although a similar limiting value of $d_{x} \approx 1.7 D_{90}$ has been observed using large tracer gravel installations on a gravel-bed river [Wilcock et al., 1996a].

\section{Conclusions}

Grains of a single size exposed on the surface of a gravel-bed river are not entrained at a single bed shear stress $\tau_{0}$ but over a range of $\tau_{0}$. Within this range, only a portion of grains exposed on the bed surface are mobilized over the duration of a transport event, a condition defined as partial transport. We demonstrate the existence and domain of partial transport using observations of grain entrainment on time series of bed photographs of flume experiments with a widely sorted sand/ gravel mixture.

For each size fraction, two populations may be identified: active grains, which are entrained at least once, and inactive grains, which remain immobile over the duration of a flume run. The proportion of active grains is shown to increase rapidly from an initial time and to asymptotically approach a 
constant value $Y_{i}$. Flow turbulence ensures that no absolute maximum proportion of active grains exists for a given size and flow, although we observe that the rate of increase of the active proportion becomes very small, approaching an effective maximum when the cumulative transport exceeds approximately 4 times the total mass of active grains. This suggests that an appropriate dimensionless timescale for bed adjustment may be defined using the ratio of cumulative transported mass to the total mass of active grains on the bed. Such a timescale provides a physical basis for estimating an appropriate averaging time for sampling entrainment and transport under conditions of partial transport.

The range of $\tau_{0}$ over which all grains in a size fraction are entrained is similar for all sizes. The transition $0.1<Y_{i}<$ 0.9 , where $Y_{i}$ is the active proportion of each fraction, occurs over a range of $\tau_{0}$ of approximately 2 for all sizes. The value of $\tau_{0}$ that entrains half of the grains in a size fraction increases with grain size such that for a given flow, grains in a state of partial transport fall within a size range of approximately a factor of 2.

A decrease in transport rate with grain size is commonly observed in gravel-bed rivers and can be directly related to conditions of partial transport. Fractional transport rates are independent of grain size for the smaller, fully mobile fractions and decrease rapidly with grain size for the larger, partially mobile fractions. A small but measurable fractional entrainment $\left(Y_{i}=0.1\right)$ is associated with vanishingly small transport rates for the coarser sizes in a mixture. Fully mobilized transport $\left(Y_{i}=1\right)$ occurs at and above a transport rate that is close to, but probably slightly smaller than, that producing equal mobility, for which the proportion of a fraction in transport becomes equal to its proportion on the bed surface. The onset of fully mobile and equally mobile transport occurs at nearly the same transport rate or shear stress for the coarsest fractions in the mixture, whereas equally mobile transport occurs at a somewhat larger flow than fully mobilized transport for fractions near the middle of the bed size distribution. Mobilization of these midsize fractions occurs in the presence of nearly complete immobility of the coarser fractions, suggesting that the gap between fully mobile and equally mobile transport results from a portion of the mobilized finer grains becoming trapped among immobile coarse grains over the duration of a transport event, thereby decreasing their proportion in the measured transport rate.

Partial transport is a grain-scale representation of incipient grain motion, in contrast to the reference shear stress $\tau_{r i}$, which is the shear stress that produces a small reference transport rate and serves as a surrogate for the critical shear stress for incipient motion. Partial transport depends on the entire population of grains present on the bed surface, whereas $\tau_{r i}$ depends on the rate of transport, regardless of grain origin, and may be produced by only a small proportion of the grains exposed on the bed surface. We observe that sufficient transport to produce the reference transport rate occurs under conditions of partial transport and that the active proportion of a fraction at the reference transport rate decreases with grain size. Both $\tau_{r i}$ and the stress mobilizing half of the surface grains in a fraction increase with grain size for our experimental sediment.

Partial transport determines the active portion of the bed surface and the size of bed pockets vacated by entrained grains. Therefore it exerts a strong influence on the size-dependent exchange of grains between the bed surface and subsurface and any process driven by grain sorting, such as armoring, selective entrainment and deposition, downstream funing, and the removal or introduction of fine-grained sediment into the subsurface. The active proportion of the bed surface varies from nearly complete immobility to complete mobilization over a range of $\tau_{0}$ of a factor of approximately 4 . Within this range the active portion of the bed consists primarily of finer grains, so that the size of vacated bed pockets will have an upper bound, which limits both the rate and size distribution of grain exchange with the bed.

The thickness $d_{x}$ of the surface layer actively participating in the transport can be approximated as the size of the fraction found to be $50 \%$ mobile at a given flow. Using this approximation for our experimental sediment, $d_{x} \propto \tau_{0}^{1.5}$ and approaches a value of approximately $2 D_{90}$ when the entire bed surface is mobilized. The variation of $d_{x}$ with $\tau_{0}$ should depend on the range of $\tau_{r i}$ for different sizes in the bed and therefore should vary from sediment to sediment. For sediments with a narrow range in $\tau_{r i}, d_{x}$ should increase rapidly over a similarly narrow range in $\tau_{0}$.

Complete mobilization of the bed surface is associated with nearly equally mobile transport of the sand and gravel portions of the sediment. Partial transport of the bed surface, primarily of the smaller sizes, occurs in the presence of nonnegligible transport rates. Because flow duration increases with decreasing flow magnitude, conditions of partial transport are common in many rivers and can produce a large proportion of the annual sediment load.

Acknowledgments. Laboratory experiments with the Bed of Many Colors were supported by grant N00014-91-J-1192 from the Office of Naval Research and grant EAR-9004206 from the National Science Foundation. Analysis of the grain immobility was supported by grant EAR-9205511 from the National Science Foundation. We thank Mike Church, Dave Furbish, Chris Paola, John Pitlick, and Ron Shreve for reviewing an earlier draft of this paper; their energetic and perceptive criticisms substantially improved the clarity and directness of the presentation.

\section{References}

Carling, P., The concept of dominant discharge applied to two gravelbed streams in relation to channel stability thresholds, Earth Surf. Processes Landforms, 13, 355-367, 1988.

Church, M., and M. A. Hassan, Size and distance of travel of unconstrained clasts on a streambed, Water. Resour. Res., 28(1), 299-303, 1992.

Church, M., J. F. Wolcott, and W. K. Fletcher, A test of equal mobility in fluvial sediment transport: Behavior of the sand fraction, Water Resour. Res., 27(11), 2941-2951, 1991.

Drake, T. G., R. L. Shreve, W. E. Dietrich, P. J. Whiting, and L. B. Leopold, Bedload transport of fine gravel observed by motionpicture photography, J. Fluid Mech., 192, 193-217, 1988.

Einstein, H. A., The bedload function for sediment transport in open channel flows, Tech. Bull. 1026, Soil Conserv. Serv., U.S. Dep. of Agric., Washington, D. C., Sept., 1950.

Hassan, M. A., M. Church, and P. J. Ashworth, Virtual rate and mean distance of travel of individual clasts in gravel-bed channels, Eanth Surf. Processes Landforms, 17, 617-627, 1992.

Hirano, M., River bed degradation with armoring, Proc. Jpn. Soc. Civ. Eng., 195, 55-65, 1971.

Jackson, W. L., and R. L. Beschta, A model of two-phase bedload transport in an Oregon coast range stream, Earth Surf. Processes Landforms, 9, 517-527, 1982.

Johnston, C., Particle friction angle variability of five natural waterworked gravel mixtures, MA thesis, Univ. of Colo., Boulder, 1996.

Kondolf, G. M., and P. R. Wilcock, The flushing flow problem: Defining and evaluating objectives, Water Resour. Res., 32(8), $2589-$ $2599,1996$. 
Kuhnle, R. A., Fractional transport rates of bedload on Goodwin Creek, in Dynamics of Gravel-Bed Rivers, edited by P. Billi, R. D. Hey, C. R. Thome, and P. Tacconi, John Wiley, New York, 1992.

Leopold, L. B., Sediment size that determines channel morphology, in Dynamics of Gravel-Bed Rivers, edited by P. Billi, R. D. Hey, C. R. Thorne, and P. Tacconi, John Wiley, New York, 1992.

Lisle, T. E., Particle size variations between bed load and bed material in natural gravel bed channels, Water Resour. Res., 31(4), 1107-1118, 1995.

Parker, G., Surface-based bedload transport relation for gravel rivers, J. Hydraul. Res., 28, 417-436, 1990.

Parker, G., Selective sorting and abrasion of river gravel, I, Theory, $J$. Hydraul. Eng., 117(2), 131-149, 1991.

Parker, G., and P. C. Klingeman, On why gravel bed streams are paved, Water Resour. Res., 18, 1409-1423, 1982.

Parker, G., and A. J. Sutherland, Fluvial armor, J. Hydraul. Res., 28(5), $529-544,1990$

Parker, G., and P. R. Wilcock, Sediment feed and recirculating flumes: A fundamental difference, J. Hydraul. Eng., 119(11), 1192-1204, 1993.

Parker, G., P. C. Klingeman, and D. L. McLean, Bedload and size distribution in paved gravel-bed streams, J. Hydraul. Div. Am. Soc. Civ. Eng., 108(HY4), 544-571, 1982.

Proffitt, G. T., and A. J. Sutherland, Transport of non-uniform sediments, J. Hydraul. Res., 21(1), 33-43, 1983.

Stelczer, K., Bedload Transport: Theory and Practice, 295 pp., Water Resour. Publ., Littleton Colo., 1981.

Wilcock, P. R., Methods for estimating the critical shear stress of individual fractions in mixed-size sediment, Water Resour. Res., 24(7), 1127-1135, 1988
Wilcock, P. R., Experimental investigation of the effect of mixture properties on transport dynamics, in Dynamics of Gravel-Bed Rivers, edited by P. Billi, R. D. Hey, C. R. Thorne, and P. Tacconi, pp. 109-139, John Wiley, New York, 1992.

Wilcock, P. R., The critical shear stress of natural sediments, J. Hydraul. Eng., 119(4), 491-505, 1993.

Wilcock, P. R., Sediment maintenance flows: Feasibility and basis for prescription, in Gravel-Bed Rivers in the Environment, edited by P. Klingeman et al., Water Resour. Press, Littleton, Colo., in press, 1996.

Wilcock, P. R., The components of fractional transport rate, Water Resour. Res., this issue.

Wilcock, P. R., and B. W. McArdell, Surface-based fractional transport rates: Mobilization thresholds and partial transport of a sand-gravel sediment, Water Resour. Res., 29(4), 1297-1312, 1993.

Wilcock, P. R., A. F. Barta, C. C. Shea, G. M. Kondolf, W. V. G. Matthews, and J. C. Pitlick, Observations of flow and sediment entrainment on a large gravel-bed river, Water Resour. Res., 32(9), 2897-2909, 1996a.

Wilcock, P. R., G. M. Kondolf, W. V. G. Matthews, A. F. Barta, Specification of sediment maintenance flows for a large gravel-bed river, Water Resour. Res., 32(9), 2911-2921, $1996 \mathrm{~b}$.

B. W. McArdell and P. Wilcock, Department of Geography and Environmental Engineering, Johns Hopkins University, Baltimore, MD 21218. (e-mail: Wilcock@jhu.edu)

(Received April 15, 1996; revised August 26, 1996; accepted August 28, 1996.) 